\title{
INIBIÇÃO DE LODO BIOLÓGICO ANAERÓBIO POR CONSTITUINTES DE EFLUENTE DE LABORATÓRIO DE CONTROLE DE POLUIÇÃO
}

\section{INHIBITION OF ANAEROBIC BIOLOGICAL SLUDGE BY EFFLUENT CONSTITUENTS OF POLLUTION CONTROL LABORATORY}

\author{
LARISSA DE CARVALHO ALVES \\ Engenheira Civil. M.Sc. em Engenharia Química. Doutoranda em Tecnologia de Processos Químicos e \\ Bioquímicos, Escola de Química, UFRJ

\section{Magali Christe CAMMARota} \\ Engenheira Química. D.Sc. em Bioquímica. Professora Adjunta do Departamento de Engenharia \\ Bioquímica, Escola de Química, UFRJ

\section{FRANCISCA PESSOAA DE FRANÇA} \\ Farmacêutica Química. D.Sc. em Microbiologia. Professora Adjunta do Departamento de Engenharia \\ Bioquímica, Escola de Química, UFRJ
}

Recebido: 16/12/04 Aceito: 12/07/05

\section{RESUMO}

Nos laboratórios de controle de poluição, análises de rotina para a caracterização de efluentes e monitoramento dos reatores de tratamento empregam soluções concentradas de $\mathrm{H}_{2} \mathrm{SO}_{4}$, contendo metais pesados como cromo, prata e mercúrio, e compostos orgânicos como fenol. Embora os microrganismos metanogênicos possam tolerar a presença de algumas substâncias ou elementos tóxicos presentes em efluentes, os mesmos podem apresentar sensibilidade a determinados compostos químicos. O trabalho teve como objetivo caracterizar o efluente do Laboratório de Tecnologia Ambiental da Escola de Química da Universidade Federal do Rio de Janeiro (LTA/UFRJ) e determinar quais constituintes desse efluente seriam inibitórios para microrganismos anaeróbios no tratamento do esgoto gerado no campus. O efluente apresenta uma produção média semanal de 43,4 L, com $\mathrm{pH} 0,7,1350 \mathrm{mg} / \mathrm{L}$ de DQO, $33500 \mathrm{mg} \mathrm{SO}_{4}^{2-} / \mathrm{L}$; 28,2 $\mathrm{mg} \mathrm{Hg} / \mathrm{L} ; 82,1 \mathrm{mg} \mathrm{Cr}_{\text {total }} / \mathrm{L}$; 30,8 $\mathrm{mg} \mathrm{Cu} / \mathrm{L}$; $57,4 \mathrm{mg} \mathrm{Fe}$ total $/ \mathrm{L} ; 16,2 \mathrm{mg} \mathrm{Al} / \mathrm{L}$ e 2,44 g Na/L. Os parâmetros analisados apresentaram elevada variabilidade ao longo do estudo, em função das análises e pesquisas realizadas naquele período. No planejamento experimental realizado, observou-se que os principais efeitos sobre o percentual de inibição da atividade metanogênica específica (AME) foram os elementos sódio, cromo, fenol e sulfato e as interações sulfato-mercúrio e sulfatosódio. Os efeitos dos demais elementos (cobre, mercúrio, alumínio e ferro), analisados separadamente e em interaçōes, não foram significativos para um nível de confiança de $95 \%$ na distribuição t de Student. Os resultados apontam para a necessidade de um tratamento específico para os efluentes de laboratório, já que as Universidades devem ser exemplos de combate à poluição e de incentivo ao controle ambiental.

PALAVRAS-CHAVES: Efluentes de laboratório, sulfatos, metais pesados, controle de poluição.

\section{ABSTRACT}

In the pollution control laboratories, routine analyses for the characterization of effluents and reactors monitoring use concentrated solutions of $\mathrm{H}_{2} \mathrm{SO}_{4}$ containing heavy metals such as chromium, silver and mercury and organic compounds as phenol. The presence of these elements in the laboratory effluent can cause inhibition of the biological activity, especially in the anaerobic treatment processes due to the sensitivity of the methanogens to some chemical compounds. This work had as objective to characterize the effluent from the Federal University of Rio de Janeiro Chemistry School Environmental Technology Laboratory (LTA/UFRJ) and to determine which constituents of the effluent one would be inhibitors for anaerobic microorganisms in the treatment of the sewage generated in the campus. The effluent one presents a weekly average production of $43.4 \mathrm{~L}$, with $\mathrm{pH}=0.7$, $C O D=1350 \mathrm{mg} / \mathrm{L}$, sulfate $=33500 \mathrm{mg} / \mathrm{L} ; 28.2 \mathrm{mg} \mathrm{Hg} / \mathrm{L}$; $82.1 \mathrm{mg} \mathrm{Cr}$ total $/ \mathrm{L} ; 30.8 \mathrm{mg} \mathrm{Cu} / \mathrm{L} ; 57.4 \mathrm{mg} \mathrm{Fe} e_{\text {total }} / \mathrm{L} ; 16.2 \mathrm{mg} \mathrm{Al/L}$ and $2.44 \mathrm{gNa} / \mathrm{L}$. The parameters analyzed presented high variability as the study was conducted in function of the analyses and researches carried out in that period. In experimental design performed, the elements sodium, chromium, phenol and sulfate, as well as the interactions sulfate-mercury and sulfate-sodium, were significantly more effective on the inhibition of the specific methanogenic activity (SMA). The effects of the other elements (cupper, mercury, aluminum and iron), analyzed alone and their interactions were not significant for a confidence level of $95 \%$ in the $t$-Student distribution. The results indicate the necessity of a specific treatment for the laboratory effluent, since universities must be examples of combating pollution and encouraging the environmental control.

KEYWORDS: Laboratory effluents, sulfate, heavy metals, environmental control. 


\section{INTRODUÇÃO}

Com as exigências do mercado e da comunidade para que as indústrias tenham comportamentos transparentes em relação às questôes ambientais, as Universidades passaram a ser solicitadas para o desenvolvimento de pesquisas ligadas ao tratamento de resíduos industriais, à descontaminação de solos e águas subterrâneas e à redução de emissōes atmosféricas. Porém, ao conduzir tais pesquisas, as instituiçôes tornaram-se potencialmente poluidoras, pois a gestão dos resíduos gerados nas Universidades e Centros de pesquisa praticamente inexiste, sendo os mesmos descartados inadequadamente (Jardim, 1998). Assim, surgiu a preocupação com o destino dado aos efluentes gerados nos laboratórios das universidades, já que as mesmas devem ser exemplos de combate à poluição e de incentivo ao controle ambiental (Benatti et al, 2004; Alves, 2002).

Praticamente todo experimento de laboratório emprega reagentes químicos e gera algum tipo de resíduo como, por exemplo, materiais de filtração, soluções ácidas e alcalinas, solventes orgânicos e substâncias químicas perigosas (como metais pesados). Além disso, a manipulação de diferentes tipos de resíduos acarreta elevadas concentrações de matéria orgânica nos efluentes dos laboratórios. Como conseqüência, tais efluentes possuem características bastante agressivas, como $\mathrm{pH}$ extremamente baixo, altos valores de Demanda Química de Oxigênio (DQO), metais pesados e sulfato.

Um novo conceito de prevenção e controle de poluição, que afeta o trabalho em laboratório, é baseado no gerenciamento de resíduos o qual incorpora uma hierarquia de técnicas de controle de poluição (NRC, 1995). Uma primeira forma de controle é a "redução na fonte", utilizando uma quantidade menor de material, materiais menos perigosos ou tornando o processo mais eficiente. Dessa forma, a substituição de um reagente químico perigoso por um não-perigoso (ou, no mínimo, menos perigoso) pode ser feita em conjunto com quantidades reduzidas do material necessário para a realização do experimento. Um exemplo de redução da quantidade de reagentes é a utilização de equipamentos analíticos como cromatógrafos, espectrofotômetros e sistemas de ressonância magnética nuclear, que requerem quantidades de material extremamente pequenas para se conduzir as análises (DPPEA, 2003).
Outro exemplo é a gestão de resíduos em uma instituição acadêmica através de uma adequada minimização e destino dos materiais gerados por cada departamento. Para atingir este objetivo, é necessário avaliar os aspectos econômicos e científicos, conscientizar e educar ambientalmente os alunos (Barbosa et al, 2003).

Entretanto, pode ocorrer de, apesar da redução na fonte ser altamente desejável, nem sempre esta alternativa é técnica e economicamente viável. Um segundo passo seria a "reciclagem/reuso/recuperação" de materiais presentes nos resíduos (NRC, 1995). Este processo requer adição de energia como, por exemplo, a destilação ou a rotoevaporação de solventes orgânicos que podem ser reutilizados. $\mathrm{O}$ terceiro passo seria o "tratamento", alternativa recomendada para materiais que não apresentam potencial para recuperação e quando não existe a possibilidade de redução na fonte. Alguns tipos de técnicas de tratamento são: neutralização, separação, fixação, oxidação, precipitação, degradação ou troca iônica (Freeman, 1997).

A última alternativa seria a "disposição no solo". Alguns resíduos perigosos, especialmente aqueles que contêm metais pesados, não podem tornar-se completamente não perigosos nem ser recuperados. Entretanto, eles precisam ser estabilizados, para reduzir a probabilidade de dispersão no ambiente, antes da disposição no solo ser permitida. Atualmente, este processo é utilizado somente em situaçōes e locais especializados e em raras ocasióes (NRC, 1995).

Das quatro alternativas apresentadas, a "redução na fonte" e a "reciclagem/reuso/recuperação" são as mais utilizadas nos laboratórios de controle de poluição. O "tratamento" quase não é aplicado pelos responsáveis pela gerência dos resíduos gerados, sendo estes segregados, armazenados e transportados para fora do local de trabalho, não havendo muita preocupação com a forma de tratamento ou disposição dos mesmos. Cabe ressaltar que na grande maioria das instituições, ainda não existe uma consciência ambiental por parte dos pesquisadores, predominando o descarte dos resíduos no lixo comum ou na rede de esgoto local, sem qualquer tratamento prévio. Tal procedimento pode afetar seriamente a eficiência das estações de tratamento de esgoto, tendo em vista que muitos constituintes desses resíduos são potencialmente tóxicos para a flora microbiana atuante nos sistemas biológicos.
Dentro deste contexto, o presente trabalho teve como objetivo caracterizar, em termos de volume produzido e composição química, o efluente oriundo de determinações analíticas diversas e da operação de reatores biológicos no Laboratório de Tecnologia Ambiental da Escola de Química da Universidade Federal do Rio de Janeiro (LTA/UFRJ). Com base na caracterização obtida, determinouse quais constituintes desse efluente seriam inibitórios para microrganismos anaeróbios no tratamento do esgoto gerado no campus. A finalidade deste estudo preliminar é obter informaçōes sobre tais efluentes para avaliar, posteriormente, o efeito de sua descarga na rede de esgoto e, consequentemente, na eficiência da estação de tratamento da Universidade ou do município.

\section{METODOLOGIA}

\section{Coleta e caracterização do efluente}

Diversas análises para a caracterização de efluentes industriais e monitoramento de reatores biológicos são efetuadas no LTA/UFRJ, utilizando uma grande variedade de reagentes, tais como ácidos, bases, sais na forma de cloretos, sulfatos e fosfatos, cromatos e dicromatos, solventes orgânicos, surfactantes aniônicos, fenol e outros (indicadores ácidobase e de oxi-redução). Estes compostos provavelmente são os principais constituintes dos efluentes gerados, juntamente com os derivados formados nas análises.

Durante cinco meses, o efluente gerado a cada semana no LTA/UFRJ foi armazenado em um recipiente de $50 \mathrm{~L}$, juntamente com a água residual da tríplice lavagem das vidrarias utilizadas nos experimentos do laboratório. Ao final de cada semana, media-se o volume de efluente e coletavam-se alíquotas para realização de análises físico-químicas. Todas as alíquotas foram adequadamente preservadas, de acordo com as análises a serem realizadas. Em um tanque com capacidade de $1000 \mathrm{~L}$, foram acumulados os efluentes gerados durante 12 semanas, sendo caracterizada também esta mistura final.

A caracterização do efluente gerado a cada semana e da mistura final foi realizada através das determinações de: $\mathrm{pH}$, Demanda Química de Oxigênio (DQO), Demanda Bioquímica de Oxigênio (DBO), turbidez, fenóis totais, óleos e graxas, sólidos suspensos totais (STS), 
Nitrogênio Kjeldahl Total (NKT), fósforo total, sódio e metais pesados $(\mathrm{Hg}, \mathrm{Cr}$, $\mathrm{Cu}, \mathrm{Ag}, \mathrm{Fe}$ e $\mathrm{Al})$.

\section{Determinação da atividade metanogênica específica (AME) do lodo biológico}

O lodo empregado como inóculo nos experimentos foi proveniente de um reator anaeróbio de fluxo ascendente em operação numa indústria alimentícia, possuindo $8296 \mathrm{mg}$ SVS/L. Os ensaios para a determinação da AME deste lodo biológico foram desenvolvidos utilizando-se frascos tipo penicilina de $100 \mathrm{~mL}$, contendo $80 \mathrm{~mL}$ de meio basal e $10 \mathrm{~mL}$ de lodo. $\mathrm{O}$ meio basal adicionado aos frascos foi composto de esgoto doméstico bruto (coletado na Estação de Tratamento de Esgoto da Ilha do Governador/RJ), solução de ácidos orgânicos voláteis (acético:propiônico:butírico - 1:1:1, em massa) e solução nutriente composta em $\mathrm{g} / \mathrm{L}$ de 3,4 $\mathrm{NH}_{4} \mathrm{Cl}, 0,7\left(\mathrm{NH}_{4}\right)_{2} \mathrm{SO}_{4} \mathrm{e}$ $0,6 \mathrm{KH}_{2} \mathrm{PO}_{4}$. Em cada frasco, adicionou-se 79,6 mL de esgoto doméstico bruto, $0,2 \mathrm{~mL}$ de solução de ácidos e $0,2 \mathrm{~mL}$ da solução nutriente, baseando-se nas relações DQO : $\mathrm{N}=120$ e DQO : $\mathrm{P}=852$, e $10 \mathrm{~mL}$ de lodo, com a finalidade de se obter uma relação inicial DQO:SVS de 1:1 (Barbosa, 1988).

Após a preparação do substrato, o $\mathrm{pH}$ foi ajustado para 7,0 com bicarbonato de sódio e os frascos foram então incubados a $35 \pm 1^{\circ} \mathrm{C}$ por 8 dias, sendo o volume de biogás produzido monitorado diariamente. $\mathrm{O}$ volume de biogás foi medido por meio de seringas graduadas e a porcentagem de metano por cromatografia gasosa com detector de condutividade térmica (TCD), em equipamento Shimadzu, modelo GC - 17A.

A conversão de volume de metano em massa de DQO foi feita de acordo com a equaçãa 1 (Chernicharo, 1997).

$\mathrm{V}_{\mathrm{CH}_{4 \mathrm{i}}}=\frac{\mathrm{DQO}_{\mathrm{CH} 4 \mathrm{i}}}{\mathrm{K}(\mathrm{t})}$

onde:

$\mathrm{V}_{\mathrm{CH} 4 \mathrm{i}}=$ volume de metano produzido no $i^{\text {ésimo }}$ dia $(\mathrm{L})$;

$\mathrm{DQO}_{\mathrm{CH} 4 \mathrm{i}}=$ massa de DQO convertida a metano no iésimo dia (gDQO);

$\mathrm{K}(\mathrm{t})$ = fator de correção para a temperatura de incubação dos frascos (gDQO/L), calculado pela Equação 2.

$\mathrm{K}(\mathrm{t})=\frac{\mathrm{P} * \mathrm{~K}}{\mathrm{R} *(273+\mathrm{t})}$

onde:

$\mathrm{P}=$ pressão atmosférica $(1 \mathrm{~atm})$;
$\mathrm{K}=\mathrm{DQO}$ correspondente $\mathrm{a}$ um mol de $\mathrm{CH}_{4}(64 \mathrm{gDQO} / \mathrm{mol})$;

$\mathrm{R}=$ constantedosgases $(0,08206 \mathrm{~atm} . \mathrm{L} / \mathrm{mol} . \mathrm{K})$; $\mathrm{t}=$ temperatura de incubação dos frascos $\left(35^{\circ} \mathrm{C}\right)$.

As AMEs foram então calculadas a partir das DQOs convertidas a metano obtidas diariamente durante o período de incubação, sendo expressas como $\mathrm{gDQO}_{\mathrm{CH} 4} / \mathrm{gSVS} . \mathrm{d}$.

\section{Atuação do lodo biológico sobre alguns constituintes do efluente do LTA/UFRJ}

O substrato foi composto de esgoto, soluçóes nutriente e de ácidos, acrescido dos elementos constituintes do efluente de laboratório em diferentes concentrações, sendo tais elementos selecionados com base na caracterização do efluente. Nestes experimentos, empregou-se um planejamento experimental fatorial fracionário $\left(2^{\mathrm{k}-4}\right)$, com dois níveis e 3 réplicas do ponto central. Com base na caracterização do efluente, foram selecionadas 8 variáveis independentes, constando de diferentes concentrações de sulfato, cobre, alumínio, prata, ferro, mercúrio, fenol e sódio, totalizando 19 experimentos, com 3 réplicas de cada condição (Tabela 1). A variável resposta adotada foi o percentual de inibição da AME do lodo biológico nas diferentes condições analisadas, calculado com base nas equaçōes 3 e 4 . A cada batelada de experimentos, foi feito o teste controle, contendo somente esgoto e soluções nutriente e de ácidos, para determinação da respectiva $\mathrm{AME}_{\text {controle }}$.

$\% \mathrm{AME}=\frac{\mathrm{AME}_{\text {teste }}}{\mathrm{AME}_{\text {controle }}} 100$

$\%$ INIB = $100-\%$ AME

A seleção dos níveis para cada variável independente analisada foi baseada em dados de toxicidade da literatura (Rinzema \& Lettinga, 1988; Spain, 2003; Speece, 1996; Vance, 2002) e nas concentraçôes obtidas na caracterização do efluente. No caso do sulfato, as concentrações adotadas não foram baseadas no efeito isolado do sulfato, tendo-se levado em consideração a relação DQO/sulfato do meio de cultivo. Em geral, efluentes com relação DQO/sulfato acima de 0,67 contêm matéria orgânica suficiente para reduzir completamente o sulfato e promover uma eficiente remoção de DQO e sulfato do efluente (Hulshoff et al, 2001). Como a DQO inicial nos experimentos era em torno de
$800 \mathrm{mg} / \mathrm{L}$, adotou-se valores mínimo e máximo de sulfato de 0,5 e $1,5 \mathrm{~g} / \mathrm{L}$, de modo que o intervalo obtido para a relação DQO/sulfato $(0,5-1,6)$ abrangesse o valor mínimo recomendado na literatura $(0,67)$. Os resultados do planejamento foram analisados através do software Statistica 5.5 para determinação das variáveis e/ou interaçôes mais significativas.

\section{Determinações quantitativas}

Demanda Química de Oxigênio (DQO), Demanda Bioquímica de Oxigênio (DBO), turbidez, fenóis totais, óleos e graxas, sólidos suspensos totais (STS), Nitrogênio Kjeldahl Total (NKT) e fósforo total foram determinados de acordo com o Standard Methods (Greenberg et al, 1992). Os metais pesados (mercúrio, cromo total, cobre, prata, ferro total e alumínio) foram quantificados através de absorção atômica, utilizando equipamento marca Micronal, modelo B262 e o elemento sódio através de fotometria de chama, utilizando o equipamento marca Perkin-Elmer, modelo 5000 . O pH foi medido por potenciometria em um medidor de pH DL - 14 marca Actron, previamente calibrado com soluções-padrão de $\mathrm{pH}=4,0$ e $\mathrm{pH}=7,0$.

\section{RESULTADOS E DISCUSSÂO}

\section{Caracterização do efluente}

Durante 12 semanas, foram realizadas as coletas semanais e caracterização dos efluentes gerados durante as atividades em andamento no LTA/UFRJ. Na Tabela 2 estão apresentados os resultados obtidos na caracterização físico-química dos efluentes semanais obtidos no período estudado. Percebe-se pela ampla faixa de variação que o efluente apresentou elevada variabilidade ao longo do tempo de amostragem, em função das características sazonais do laboratório. Esta sazonalidade está relacionada com mudanças nas linhas de pesquisa e, portanto, nos trabalhos e análises desenvolvidos nos laboratórios.

Os valores de $\mathrm{pH}$ dos efluentes semanais gerados ao longo do estudo foram extremamente baixos $(<1,0)$, provavelmente devido à grande quantidade de ácidos utilizados nas análises desenvolvidas no laboratório, principalmente ácido sulfúrico. Além disso, obteve-se altas concentrações de sulfato em função de pes- 
quisas conduzidas com efluentes da área de petróleo (águas produzidas de plataformas offshore) e realização de grande quantidade de análises de DQO (cujas soluções reagentes são formuladas com ácido sulfúrico) e matéria orgânica (que empregam sulfato ferroso amoniacal como reagente). Neste efluente também foram encontradas altas concentrações de fenol devido à determinação de NKT por método colorimétrico, que emprega fenol como reagente. Vale ressaltar a baixa relação $\mathrm{DBO}_{5} / \mathrm{DQO}(<0,01)$ obtida para este efluente, o que indica a presença de compostos persistentes e/ou inibidores do metabolismo microbiano. Os metais pesados ( $\mathrm{Hg}, \mathrm{Cr}, \mathrm{Cu}, \mathrm{Ag}, \mathrm{Fe}$ e $\mathrm{Al})$ e o sódio foram quantificados apenas na mistura final, apresentando elevadas concentraçōes, com exceção da prata.

Alves (2002) caracterizou o efluente do Laboratório de Tratamento de Efluentes da Universidade Federal de Uberlândia (LTE/UFU), obtendo altas concentrações de sólidos suspensos totais $(4208 \pm 1311 \mathrm{mg} / \mathrm{L})$, DQO $(6535 \pm 3165 \mathrm{mg} / \mathrm{L})$, óleos e graxas $(408,9 \pm 111,9 \mathrm{mg} / \mathrm{L})$ e NKT $(295,5 \pm 38,4 \mathrm{mg} / \mathrm{L})$. Este efluente continha grandes quantidades de soro de queijo não aproveitado, pois no período estudado este laboratório realizava várias pesquisas com efluentes de laticínio, acarretando elevadas concentraçóes de gordura (óleos e graxas) e proteínas no mesmo. As gorduras, em geral, apresentamse como partículas insolúveis, contribuindo também para os altos teores de sólidos suspensos. Já as proteínas, resultavam em elevados teores de NKT no efluente.

Benatti et al. (2004) estudaram o efluente do Laboratório de Controle de Poluição da Universidade Estadual de Maringá (LCP/UEM) obtendo altas concentrações de fenóis totais (34,2-784,6 mg/L), cálcio (4,9-112,0 mg/L), sódio $(824,2-8237,8 \mathrm{mg} / \mathrm{L})$ e potássio (182,3 - 918,8 mg/L). Com relação aos metais analisados, a concentração de cromo total neste efluente foi bem maior que nos efluentes gerados no LTE/UFU e no LTA/UFRJ, variando numa faixa entre 157,5 e 589,6 mg/L. Tais características provavelmente são consequência das diferentes tipologias industriais estudadas neste laboratório, que no período analisado realizava experimentos com efluentes de manipueiras e indústrias de gelatina.

Os três efluentes mencionados apresentaram algumas características em comum, como $\mathrm{pH}$ extremamente baixo $(<1,0)$, altos valores de turbidez $(>200$

Tabela I - Concentrações mínima, média e máxima adotadas para cada constituinte selecionado e o respectivo reagente utilizado em cada caso

\begin{tabular}{cccr}
\hline Variáveis & \multicolumn{3}{c}{ Concentração $(\mathrm{mg} / \mathrm{L})$} \\
& Mínima & Média & Máxima \\
\hline Sulfato $\left(\mathrm{Na}_{2} \mathrm{SO}_{4}\right)$ & 500,0 & 1000 & 1500,0 \\
Fenóis totais $(\mathrm{Fenol})$ & 50,0 & 150 & 250,0 \\
Ferro $\left(\mathrm{FeSO}_{4}\right)$ & 12,0 & 36 & 60,0 \\
Cobre $\left(\mathrm{CuSO}_{4}\right)$ & 0,5 & 15 & 29,5 \\
Alumínio $\left(\mathrm{Al}_{2}\left(\mathrm{SO}_{4}\right)_{3}\right)$ & 0,5 & 8 & 15,5 \\
Mercúrio $\left(\mathrm{HgSO}_{4}\right)$ & 2,0 & 15 & 28,0 \\
Cromo $\left(\mathrm{K}_{2} \mathrm{Cr}_{2} \mathrm{O}_{7}\right)$ & 20,0 & 50 & 80,0 \\
Sódio $(\mathrm{NaCl})$ & 500,0 & 2500 & 4500,0 \\
\hline
\end{tabular}

Tabela 2 - Caracterização físico-química dos efluentes gerados semanalmente no LTA/UFRJ

\begin{tabular}{|c|c|c|}
\hline Parâmetro $^{a}$ & Faixa de variação & Valor médio \\
\hline $\mathrm{pH}$ & $0,6-0,9$ & 0,7 \\
\hline Turbidez (FTU) & $80-2500$ & 826 \\
\hline Sólidos Suspensos Totais & $36-1368$ & 502 \\
\hline DQO & $1000-5900$ & 1908 \\
\hline $\mathrm{DBO}_{5}$ & $12-40$ & 21 \\
\hline Sulfato & $15300-81000$ & 36700 \\
\hline Cloreto & $428-26410$ & 3915 \\
\hline Óleos e Graxas & $0,0-220$ & 46 \\
\hline Fenóis totais & $0,4-326,8$ & 132,1 \\
\hline Nitrogênio Kjeldahl Total & $0,1-38,3$ & 17,4 \\
\hline Fósforo Total & $0,0-691,8$ & 95,5 \\
\hline Sódio & $2440,0^{b}$ & - \\
\hline Mercúrio & $28,2^{b}$ & - \\
\hline Cromo total & $82,1^{b}$ & - \\
\hline Cobre & $30,8^{b}$ & - \\
\hline Prata & $0,1^{\mathrm{b}}$ & - \\
\hline Ferro total & $57,4^{\mathrm{b}}$ & - \\
\hline Alumínio & $16,2^{b}$ & - \\
\hline Volume Acumulado por Semana (L) & $24,7-63,0$ & 43,4 \\
\hline Volume Total Acumulado (L) & 521,2 & - \\
\hline
\end{tabular}

${ }^{a}$ todos os parâmetros estão em $\mathrm{mg} / \mathrm{L}$, exceto aqueles indicados na tabela; ${ }^{\mathrm{b}}$ valor relativo à mistura de todos os efluentes gerados semanalmente.

FTU), e elevadas concentrações de DQO (>1350 mg/L), sulfato (>10 g/L), sódio (>800 mg/L), ferro total (> $57 \mathrm{mg} / \mathrm{L})$ e alumínio $(>16 \mathrm{mg} / \mathrm{L})$.

\section{Determinação da atividade metanogênica (AME) do lodo biológico}

A Figura 1, a título de exemplo, mostra uma curva representativa da AME do lodo biológico ao longo do tempo. 
Neste exemplo é apresentada a curva média dos resultados obtidos, de acordo com metodologia adotada por Barbosa (1988), para cinco amostragens de esgotos sem adição de nenhum dos constituintes do efluente selecionados como potencialmente tóxicos para a flora microbiana. Observa-se que a AME aumentou até o $5^{\circ}$ dia de incubação, estabilizando a partir deste dia. Com base nas várias curvas obtidas ao longo do estudo, levantadas para cada batelada de ensaios realizados, determinou-se a produtividade máxima de metano ou AME máxima, que permaneceu na faixa de 0,10 - 0,16 gDQO/gSVS.d. Os valores obtidos no presente estudo mostraram-se compatíveis com os valores citados na literatura: 0,17 - 0,23g DQO/gSVS.d (Barbosa, 1988), 0,17-0,25 gDQO/gSVS.d(Lettinga, 1982) e $0,10-0,25 \mathrm{gDQO} / \mathrm{gSVS} . \mathrm{d}$ (Schellinkhout, 1985). Os menores valores obtidos provavelmente foram em decorrência do maior acúmulo de sólidos no lodo, originários do esgoto doméstico bruto utilizado. Com base nestas observaçōes, pode-se concluir que os microrganismos presentes no lodo apresentavam boa atividade metanogênica, sendo adequados para serem usados no tratamento do efluente gerado no LTA/UFRJ.

\section{Atuação do lodo biológico sobre alguns constituintes do efluente do LTA/UFR J}

O planejamento fatorial fracionário é apresentado na Tabela 3, juntamente com os resultados obtidos para o percentual de inibição. O software usado gerou uma curva de regressão linear com coeficiente de correlaçáo de $0,9931\left(\mathrm{r}^{2}\right)$ para um intervalo de confiança de $95 \%$. Para se avaliar que variáveis eram significativas ou não sobre o percentual de inibição da AME do lodo anaeróbio, foi empregado o teste t de Student. O valor do $t$ de Student foi obtido através do número de graus de liberdade para os experimentos, sendo fornecido pelo software. Dessa forma, assumindo-se um intervalo de confiança de $95 \%$ e 3 graus de liberdade (o erro do grau de liberdade no planejamento experimental fracionário $2^{8-4}$ foi 3), tem-se um valor de t tabelado de 3,182. Com base neste valor, as variáveis significativas na inibição do consórcio microbiano anaeróbio foram analisadas. Para que uma variável ou interação seja considerada importante, o valor de $t$ calculado deve ser maior que o tabelado.

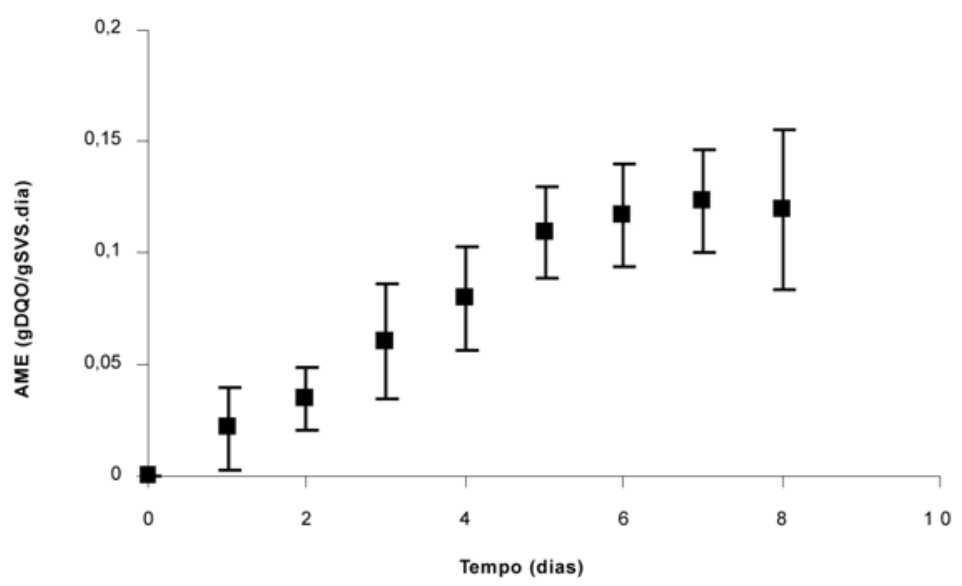

Figura I - Curva média da atividade metanogênica específica (AME) do lodo selecionado
Estas análises são melhor visualizadas num Diagrama de Pareto (Figura 2), no qual a linha vertical (para $\mathrm{p}=0,05$, o valor de $\mathrm{t}$ de Student tabelado) indica a mínima magnitude das variáveis estatisticamente significativas na análise em questão.
Na Figura 2, os valores mostrados nas colunas horizontais correspondem ao t de Student calculado para cada variável ou interação. Os efeitos positivos indicam que as variáveis consideradas apresentam efeito tóxico sobre a AME, e que
Tabela 3 - Planejamento fatorial fracionário em dois níveis com 3 réplicas do ponto central

\begin{tabular}{|c|c|c|c|c|c|c|c|c|c|}
\hline Corrida & Sulfato & Fenol & Ferro & Cobre & Alumínio & Cromo & Mercúrio & Sódio & $\begin{array}{c}\text { Inibiçāo } \\
(\%)\end{array}$ \\
\hline 8 & 1 & 1 & 1 & -1 & -1 & -1 & 1 & -1 & 15,9 \\
\hline 1 & -1 & -1 & -1 & -1 & -1 & -1 & -1 & -1 & 1,9 \\
\hline 9 & -1 & -1 & -1 & 1 & 1 & 1 & -1 & 1 & 92,4 \\
\hline 17 & 0 & 0 & 0 & 0 & 0 & 0 & 0 & 0 & 44,4 \\
\hline 7 & -1 & 1 & 1 & -1 & -1 & 1 & -1 & 1 & 96,2 \\
\hline 6 & 1 & -1 & 1 & -1 & 1 & -1 & -1 & 1 & 4,1 \\
\hline 3 & -1 & 1 & -1 & -1 & 1 & -1 & 1 & 1 & 72,6 \\
\hline 14 & 1 & -1 & 1 & 1 & -1 & 1 & -1 & -1 & 0,0 \\
\hline 5 & -1 & -1 & 1 & -1 & 1 & 1 & 1 & -1 & 0,0 \\
\hline 16 & 1 & 1 & 1 & 1 & 1 & 1 & 1 & 1 & 100,0 \\
\hline 4 & 1 & 1 & -1 & -1 & 1 & 1 & -1 & -1 & 0,0 \\
\hline 12 & 1 & 1 & -1 & 1 & -1 & -1 & -1 & 1 & 29,1 \\
\hline 15 & -1 & 1 & 1 & 1 & 1 & -1 & -1 & -1 & 20,4 \\
\hline 19 & 0 & 0 & 0 & 0 & 0 & 0 & 0 & 0 & 39,8 \\
\hline 10 & 1 & -1 & -1 & 1 & 1 & -1 & 1 & -1 & 0,0 \\
\hline 2 & 1 & -1 & -1 & -1 & -1 & 1 & 1 & 1 & 52,5 \\
\hline 18 & 0 & 0 & 0 & 0 & 0 & 0 & 0 & 0 & 41,1 \\
\hline 13 & -1 & -1 & 1 & 1 & -1 & -1 & 1 & 1 & 44,7 \\
\hline 11 & -1 & 1 & -1 & 1 & -1 & 1 & 1 & -1 & 22,8 \\
\hline
\end{tabular}

* (1) = Concentração máxima; $(-1)$ = Concentração mínima; $(0)$ = Concentração média. 
o aumento da concentração resulta em mais inibição. Os efeitos negativos indicam que o aumento da concentração das variáveis resulta em menor inibição. As interações também podem ser positivas ou negativas. Uma interação sulfato-sódio negativa (como mostrado na Figura 2) indica que ao se mudar a variável sulfato do maior (+1) para o menor nível (-1), o efeito do sódio aumenta, e ao se mudar a variável sódio do maior nível para o menor, o efeito do sulfato aumenta. Assim, o gráfico Pareto mostra que os principais efeitos sobre o percentual de inibição da AME foram os elementos sódio, cromo, fenol e sulfato e as interaçōes sulfatomercúrio e sulfato-sódio. Os efeitos dos demais elementos (cobre, mercúrio, alumínio e ferro), analisados separadamente e em interaçôes, não foram significativos para um nível de confiança de $95 \%$ na distribuição t de Student.

Os coeficientes de cada variável e interação do modelo matemático gerado pelo software para o planejamento experimental fracionário são apresentados na Tabela 4, assim como os valores dos efeitos (t de Student calculado) e seus respectivos erros padrōes. Os efeitos são calculados dividindo-se o coeficiente de cada variável ou interação pelo respectivo erro padrão fornecido pelo software.

Portanto, pode-se dizer que quanto maior a concentração de sódio, cromo ou fenol, maior a inibição do lodo biológico. No caso do sulfato, quanto maior a concentração do mesmo no meio, menor a inibição do lodo biológico. No entanto, o valor da porcentagem de inibição para o sulfato é praticamente o mesmo, tanto no nível mínimo como no máximo (Figura 3). Além disso, é importante ressaltar que a concentração de sulfato existente no efluente $(33500 \mathrm{mg} / \mathrm{L})$ é muito superior aos níveis mínimo $(500 \mathrm{mg} / \mathrm{L})$ e máximo (1500 mg/L) adotados no planejamento experimental, o que não acontece com os outros elementos significativos, cujos valores de concentração no efluente estão dentro do intervalo de níveis mínimo e máximo estudados.

Deve-se considerar que os resultados obtidos na pesquisa são apenas indicativos, pois os efeitos do descarte dos efluentes gerados nos laboratórios na etapa de tratamento biológico variarão não só com as diferentes análises realizadas nos diversos laboratórios como também com o lodo e as diluiçōes (do efluente na rede de esgoto) utilizados. No caso do lodo, dependendo do reator e da água residuária tratada, os resultados certamen-

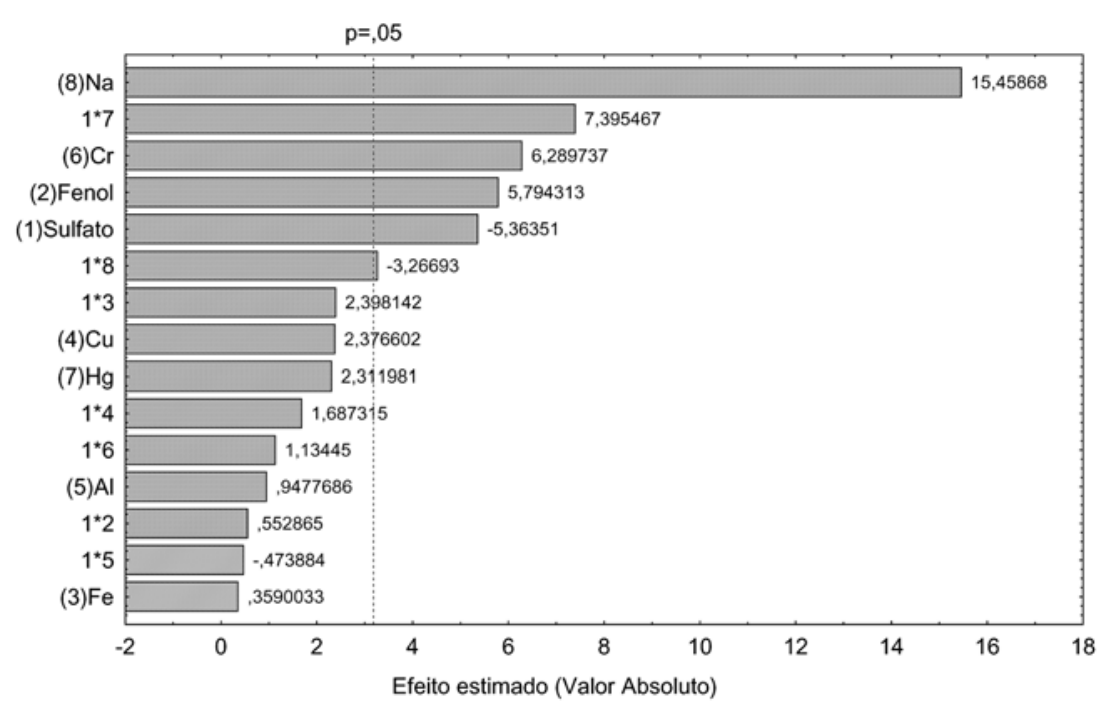

Figura 2 - Diagrama de Pareto: I sulfato; 2 fenol; 3 ferro; 4 cobre; 5 alumínio; 6 cromo; 7 mercúrio; 8 sódio

\begin{tabular}{|c|c|c|c|}
\hline Variável/Interação & Coeficiente & Erro padrão & Efeito \\
\hline Sulfato (1) & $-9,3375$ & 1,7409 & $-5,3635$ \\
\hline Fenol (2) & 10,0875 & 1,7409 & 5,7943 \\
\hline $\mathrm{Fe}(3)$ & 0,6250 & 1,7409 & 0,3590 \\
\hline $\mathrm{Cu}(4)$ & 4,1375 & 1,7409 & 2,3766 \\
\hline $\mathrm{Al}(5)$ & 1,6500 & 1,7409 & 0,9478 \\
\hline $\mathrm{Cr}(6)$ & 10,9500 & 1,7409 & 6,2897 \\
\hline $\mathrm{Hg}(7)$ & 4,0250 & 1,7409 & 2,3120 \\
\hline $\mathrm{Na}(8)$ & 26,9125 & 1,7409 & 15,4587 \\
\hline $1^{*} 2$ & 0,9625 & 1,7409 & 0,5529 \\
\hline $1^{*} 3$ & 4,1750 & 1,7409 & 2,3981 \\
\hline $1 * 4$ & 2,9375 & 1,7409 & 1,6873 \\
\hline $1 * 5$ & $-0,8250$ & 1,7409 & $-0,4739$ \\
\hline $1^{*} 6$ & 1,9750 & 1,7409 & 1,1345 \\
\hline $1^{*} 7$ & 12,8750 & 1,7409 & 7,3955 \\
\hline $1 * 8$ & $-5,6875$ & 1,7409 & $-3,2669$ \\
\hline
\end{tabular}

te serão diferentes dos obtidos neste trabalho. Isso ocorre devido, principalmente, às diferentes concentraçôes de organismos presentes nos diversos lodos anaeróbios e a diferente diversidade microbiana, que é dependente de vários fatores como tipo do reator, parâmetros operacionais, e tipo de água residuária, entre outros.
Apesar destas considerações, os resultados obtidos apontam para a necessidade de uma etapa de pré-tratamento anterior a etapa biológica, a fim de reduzir as concentraçôes dos elementos considerados mais tóxicos para os microrganismos. Tal medida permitiria a manutenção da eficiência das Estações de Trata- 

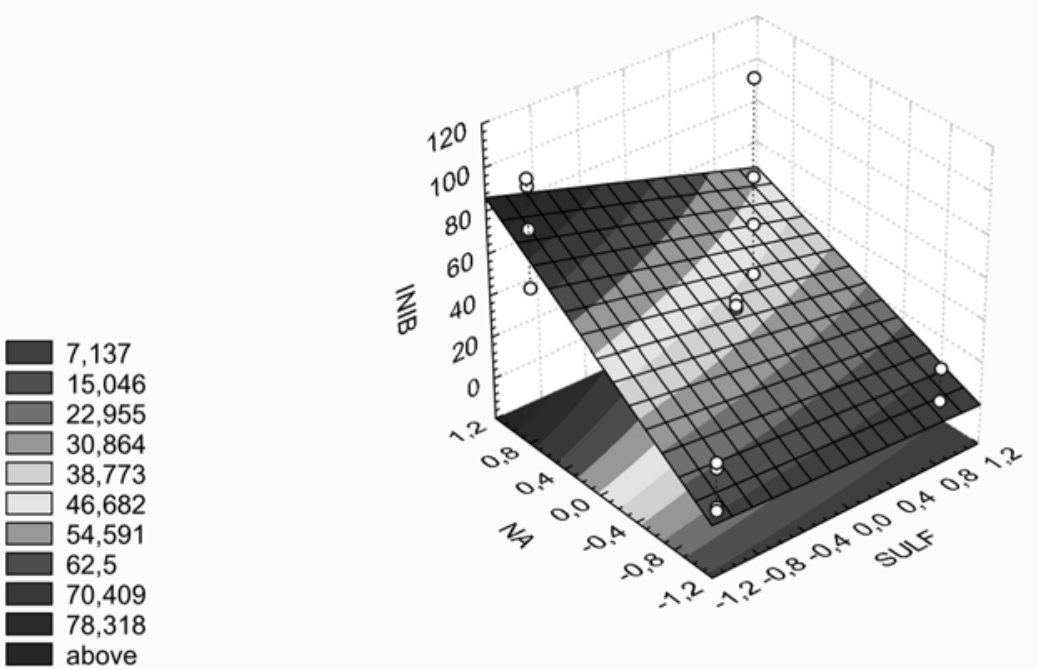

Figura 3 - Superfície de resposta de inibição da AME, mediante diferentes concentrações de sulfato e sódio, mantendo-se as outras variáveis constantes no valor médio de concentração

mento de Esgoto mediante o descarte de efluentes com altas concentraçóes de compostos tóxicos na rede de esgoto após um tratamento adequado. A eficiência de remoção destes elementos através de processos físico-químicos (precipitação química e coagulação/floculação) e eletrolíticos vem sendo avaliada no LTA/UFRJ.

\section{CONCLUSÕES}

As análises de rotina realizadas no Laboratório de Tecnologia Ambiental da Escola de Química/UFRJ geram efluentes com valores de $\mathrm{pH}$ extremamente baixos, altas concentraçôes de DQO (1908 mg/L), sulfato $(33500 \mathrm{mg} / \mathrm{L})$, metais pesados $(28,2 \mathrm{mg} \mathrm{Hg} / \mathrm{L}$; $82,1 \mathrm{mg} \mathrm{Cr}_{\text {total }} / \mathrm{L} ; 30,8 \mathrm{mg} \mathrm{Cu} / \mathrm{L}$; $57,4 \mathrm{mg} \mathrm{Fe}{ }_{\text {total }} / \mathrm{L} ; 16,2 \mathrm{mg} \mathrm{Al} / \mathrm{L} \mathrm{e}$ 2,44 g Na/L) e baixa biodegrabilidade $\left(\right.$ relação $\left.\mathrm{DBO}_{5} / \mathrm{DQO}<0,01\right)$.

Os resultados obtidos a partir do planejamento experimental fracionário mostraram que os elementos de maior efeito sobre o percentual de inibição da AME foram sódio, cromo, fenol e sulfato. Assim, deve-se promover a máxima redução possível das concentraçōes destes elementos durante o pré-tratamento do efluente, a fim de permitir um eficiente tratamento biológico posterior.

Apesar das diferenças inerentes às pesquisas e estudos desenvolvidos em cada laboratório, os resultados obtidos reforçam a necessidade de caracterização dos efluentes e o estudo de técnicas de tratamento adequadas para reduzir a toxicidade e aumentar a biodegradabilidade dos mesmos antes de sua descar- ga nas Estações de Tratamento de Esgoto das Universidades ou dos municípios.

\section{AGRADECIMENTOS}

Os autores agradecem à CAPES, CNPq, FUJB e FAPERJ pelo apoio financeiro concedido para o desenvolvimento da pesquisa.

\section{REFERÊNCIAS}

ALVES, L. C. Caracterização e tratamento de efluentes de laboratório de análises químicas. Dissertação de Mestrado, Faculdade de Engenharia Química, Universidade Federal de Uberlândia, 163p. 2002.

BARBOSA, R. Tratamento anaeróbio de esgoto sanitário em reator de fluxo ascendente com leito de lodo. Dissertação (Mestrado) - COPPE / Universidade Federal do Rio de Janeiro, Rio de Janeiro, 242p. 1988.

BARBOSA, D.P. et al. Gerenciamento de residuos dos laboratórios do Instituto de Química da Universidade do Estado do Rio de Janeiro como um projeto educacional e ambiental. Engenharia Sanitária e Ambiental, v.8, n.3, 114-9, 2003

BENATTI, C.T.; TAVARES, C.R.G.; TONIOLO, F.S. Aplicação dos sistemas $\mathrm{Fe}^{2+} / \mathrm{H}_{2} \mathrm{O}_{2}$ e $\mathrm{Fe}^{3+} / \mathrm{H}_{2} \mathrm{O}_{2}$ no tratamento de efluentes quimicos. In: CONGRESSO BRASILEIRO DE CIÊNCIA E TECNOLOGIA EM RESÍDUOS E DESENVOLVIMENTO SUSTENTÁVEL, 1, 2004, Florianópolis. Livro de Resumos, Florianópolis: ICTR, p. 98-8. 2004.

CHERNICHARO, C. A. L. Reatores Anaeróbios. Belo Horizonte: SEGRAC,. v.5, 245p. 1997.

DPPEA - N. C. Division of Pollution Prevention and Environmental Assistance, 2003. http://www.p2pays.org/Acessado em $10 / 09 / 2004$.
FREEMAN, H. M. Hazardous waste treatment and disposal. New York: McGraw-Hill Companies, 1997.

GREENBERG, A. E., CONNORS, J. J., JENKIS, D. Standard Methods for the Examination of Water and Wastewater. New York: APHA, AWWA, WPCF, 1992

HULSHOFF POL, L.W. et al. New developments in reactor and process technology for sulfate reduction. Water Science Technology, v. 44, n. 8, $67-76,2001$.

JARDIM, W.F. Gerenciamento de residuos quimicos em laboratórios de ensino e pesquisa. Química Nova, v. 21, n.5, 671-3, 1998.

LETTINGA, G. et al. Anaerobic treatment of sewage and low strenght wastewaters.In: HUGHES, D. E. et al. ANAEROBIC DIGESTION, 1981, Amsterdam. Elsevier Biomedical Press, p. 271-91. 1982.

NRC - NATIONAL RESEARCH COUNCIL. Prudent practices in the laboratory handling and disposal of chemicals. Washington, DC: National Academy Press, 1995.

RINZEMA, A.; LETTINGA, G. Anaerobic treatment of sulfate containing wastewater. In: WISE D.L. Biotreatment systems. Boca Raton, EUA: CRC Press Inc., 65 - 109, 1988.

SCHELLINKHOUT, A. et al. The application of th UASB reactor for direct treatment of domestic wastewater under tropical conditions. In: ANAEROBIC TREATMENT OF SEWAGE SEMINAR. Massachusets: Proceedings, 1985.

SPAIN, A. Implications of microbial heavy metal tolerance in the environment. Undergrad. Res., Oklahoma, EUA, v. 2, n. 1-6, 2003.

SPEECE, R. E. Anaerobic Biotechnology. Nashville: Archae Press, 394 p. 1996.

VANCE, D. 2 the 4 Technology Solutions - what's toxic, what's not. Ground water columns, 2002. http://2the4.net/toxic.htm/Acessado em: 04/ $05 / 2004$

\section{Endereço para correspondência:}

\section{Larissa de Carvalho Alves}

Universidade Federal do Rio de

Janeiro

Escola de Química - Centro de

Tecnologia

BI. E - Sala 203 Cidade Universitária 2 I $949-900$ Rio de Janeiro - RJ-

\section{Brasil}

Tel.: (2I) 2562-7624

Fax: (2I) 2562-7567

E-mail: christe@eq.ufrj.br 